\title{
The Mediterranean Diet: Plant Lectins as Essential Components
}

\author{
Emeritus Ian F Pryme* \\ Department of Biomedicine,University of Bergen, Norway \\ *Corresponding author: Emeritus Ian F Pryme, Department of Biomedicine,University of Bergen, Norway \\ To Cite This Article: Emeritus Ian F Pryme, The Mediterranean Diet: Plant Lectins as Essential Components. Am J Biomed Sci \& Res. 2020 - 7(2). \\ AJBSR.MS.ID.001126. DOI: 10.34297/AJBSR.2020.07.001126.
}

Received: 眥 January 29, 2020; Published: 拝 February 03, 2020

\section{Opinion}

In the U.S. News and World Report annual rankings (published in 2020) we note that the Mediterranean diet (Md) has, for the third year in row, been named as the overall best healthy diet. This diet is well-known for its emphasis on a high content of fruits, nuts, vegetables and whole grains. In some meals in this class of diet one can find more than 20 individual components belonging to these groups of foodstuffs. For many years now the Mediterranean way of life has been highly looked upon for health inspiration. Based on studies of Md it is now well recognised that diets rich in fruits, vegetables, fish, and healthy fats are good for us, particularly our hearts. Numerous studies have clearly shown that eating like they do in countries such as Greece, Italy, and Turkey then there is a reduced risk of suffering from heart disease. Furthermore, according to health professionals at the Mayo Clinic, those that adhere to the Md show a reduced risk of developing cancer, Parkinson's disease, and Alzheimer's disease. So, what makes the Md exceptional?

In my opinion one should look for a common factor among the ingredients of the Md. A recent survey of the content of fruits, nuts, vegetables and whole grains has shown that all these commodities contain varying amounts of protein molecules termed lectins. Health authorities in Western society strongly advise members of the population to adopt the "five a day" principle i.e. one should include at least five portions of plant-based material in the diet per day (approx. 400g). In actual fact health benefactors are thus advising us that a diet rich in lectins is beneficial to health! This is in stark contrast to what can be described as anti-lectin propaganda that we read in the popular press and in opinions expressed on numerous web sites. It is indeed correct that there are reports in the scientific literature (animal experiments) that have demonstrated negative effects of diets highly enriched in certain lectins.

The amounts added to these artificial diets fed to animals are, however, in most cases so high that it would be impossible for an individual following a normal diet to attain a lectin intake likely to be detrimental to health. Furthermore, there is no evidence that individuals that keep to a vegetarian diet, i.e. daily are exposed to a diet enriched in lectins, show any signs of poor health. So what are lectins? Plant lectins were discovered by Stillmark in 1888 when he observed that castor bean extracts caused agglutination of red blood cells in vitro. The word lectin (legere; from latin, means to select) was coined by Boyd in 1954 to cover a group of hemagglutinins that were able to discriminate between blood types in the ABO system. This hemagglutination property of lectins has been exploited as a useful method to identify the presence of lectins in, for example, food extracts. This approach was used by Nachbar and Oppenheim in 1980 when they reported that lectins were present in $>20$ common food commodities including potatoes, carrots, tomatoes, beans and peas.

Lectins are proteins that are in general resistant to low $\mathrm{pH}$ in the stomach and to proteolytic enzymes in the small intestine, and for some of these molecules $>90 \%$ can survive passage through the gut. It is therefore evident that biologically active lectins will be present in the intestine after a meal containing raw plant material. About $80 \%$ of our immune system is associated with the alimentary canal, such that dietary lectins will provide an activation of our immunedefense apparatus. One can surmise that a diet lacking active plant lectins would result in a weakened immune system. Plant lectins have physiological effects, such as binding to glycoproteins on the epithelial surface of the small intestine, where they may elicit local and/or systemic reactions, e.g. modulation of the immune system and the micro flora in the gastrointestinal tract.

That different lectins bind to specific regions of the small intestine has been clearly demonstrated in experiments using human biopsies. In 1996 Sharma and colleagues used a panel of 27 lectins to study binding to M-cells, enterocytes, goblet cells, 
lymphocytes and macrophages. They clearly established that lectins with known differences in binding properties showed individual specificity with regard to which region of the small intestine they bound. For example, only 13/27 were found to bind to the M-cells of the follicle-associated epithelium and of these 5 bound to goblet cells. Although the biological significance of this is currently not known, some lectinologists speculate that lectins which have the property of binding to different cell types associated with the small intestine will initiate or trigger, separate biological responses positive for our health/well-being. The lectin, following binding to the cell, can send information into the cell's interior via second messengers, often modulating gene expression or the lectin itself may enter the cell through endocytosis.

A large number of animal studies have clearly shown that dietary supplementation with various lectins can reduce growth of a series of different types of tumors. In their review published in 2005 Gonzalez De Mejia and Prisecaru indicate that use of plant lectins may provide novel strategies in the future for the development of new forms of cancer treatment. Beneficial effects have been reported in cases of terminal cancer where lectin-based preparations have been utilized. As mentioned earlier lectins are protein molecules meaning that they are denatured by the high temperatures met during cooking, frying, grilling etc. resulting in loss of biological activity. Thus, the importance of emphasising the need to consume raw plant material.

Dietary lectin studies are complicated by the fact that humans have consumed lectins in their habitual diet for thousands of years, making the study of individual lectins thus virtually impossible, since many may well act in concert. Furthermore, a meal would seldom consist of e.g. a single vegetable. There is indeed information indicating that when different lectin-containing preparations are mixed separately and in various proportions, then the biological responses elicited are quite unlike. Plant lectins represent an unavoidable component of a balanced diet. During the past 1020 years more and more studies have been addressed to the conception that lectin molecules may have an important positive impact on our health in general. Current opinion concerns the potential that lectins may have in relation to preventing illness and should therefore be regarded as health promoting components in our diet along with vitamins, minerals and other micronutrients.

In conclusion there can be no doubt that the Md is enriched in lectins and that these protein molecules can promote both positive and beneficial effects on our health. It is therefore not difficult to voice the opinion that a balanced diet containing a rich variety of fruits, vegetables and nuts will provide the necessary diversity of lectins required to stimulate biological processes in the body, forming the basis of a healthy lifestyle. Although there is much talk of the benefits of Md there is no information readily available to the general public on why a plant-based diet is positive for our health. For good health it is not simply a matter of obtaining sufficient amounts of nutrients from our food and vitamins and minerals etc. by an intake of tablets from the local drugstore, since lectins can only be obtained, not in the form of a pill, but by ingestion of raw plant material purchased at the supermarket! In my opinion more public awareness needs to be directed to the content of Md. 